PROCEEDINGS OF THE

AMERICAN MATHEMATICAL SOCIETY

Volume 132, Number 5, Pages 1411-1417

S 0002-9939(03)07207-1

Article electronically published on August 20, 2003

\title{
AN ENDPOINT ESTIMATE FOR THE DISCRETE SPHERICAL MAXIMAL FUNCTION
}

\author{
ALEXANDRU D. IONESCU
}

(Communicated by Andreas Seeger)

\begin{abstract}
We prove that the discrete spherical maximal function extends to a bounded operator from $L^{d /(d-2), 1}\left(\mathbb{Z}^{d}\right)$ to $L^{d /(d-2), \infty}\left(\mathbb{Z}^{d}\right)$ in dimensions $d \geq 5$. This is an endpoint estimate for a recent theorem of Magyar, Stein and Wainger.
\end{abstract}

\section{INTRODUCTION}

The discrete spherical maximal function is defined as the operator

$$
A_{*}(f)(n)=\sup _{r \in \Lambda} \frac{1}{N_{d}(r)} \sum_{|m|=r}|f(n-m)|,
$$

where $f: \mathbb{Z}^{d} \rightarrow \mathbb{C}$ is a function, $d \geq 2, N_{d}(r)$ denotes the number of lattice points on the sphere $\left\{x \in \mathbb{R}^{d}:|x|=r\right\}$ and $\Lambda=\left\{r \geq 0: N_{d}(r) \neq 0\right\}$. The sum in the definition of the operator $A_{*}$ is taken over the lattice points $m$ on the sphere of radius $r$. This operator is the discrete analogue of the classical Euclidean spherical maximal function

$$
\mathcal{A}_{*}(f)(x)=\sup _{r \in(0, \infty)}|f| * d \sigma_{r}(x)
$$

where $d \sigma_{r}$ denotes the normalized invariant measure on the sphere $|x|=r$ and $f: \mathbb{R}^{d} \rightarrow \mathbb{C}$ is a suitable function. It is well known that the operator $\mathcal{A}_{*}$ extends to a bounded operator on $L^{p}\left(\mathbb{R}^{d}\right)$ for $d \geq 2$ and $p>d /(d-1)$ (see Stein [7] in the case $d \geq 3$ and Bourgain [2] in the case $d=2$ ).

The question of boundedness on $L^{p}\left(\mathbb{Z}^{d}\right)$ of the operator $A_{*}$ was considered by Magyar [4] and Magyar, Stein and Wainger [5]. The main theorem in [5] is the following.

Theorem. (Magyar, Stein and Wainger 5). The maximal operator $A_{*}$ extends to a bounded operator on $L^{p}\left(\mathbb{Z}^{d}\right)$ if and only if $d \geq 5$ and $p>d /(d-2)$ or $d \leq 4$ and $p=\infty$.

The distinction between the cases $d \geq 5$ and $d \leq 4$ is related to the behavior of the function $N_{d}(r)$. If $d \leq 4$, this function is irregular. On the other hand, it is well known that if $d \geq 5$, then there is a constant $C_{d} \geq 1$ such that $C_{d}^{-1} r^{d-2} \leq$

Received by the editors November 11, 2002 and, in revised form, December 31, 2002.

2000 Mathematics Subject Classification. Primary 42B25.

The author was supported in part by the National Science Foundation under NSF Grant No. 0100021 .

(C)2003 American Mathematical Society 
$N_{d}(r) \leq C_{d} r^{d-2}$ for any $r \geq 1$ with the property that $r^{2} \in \mathbb{Z}$. In particular, the set $\Lambda$ in the definition of the operator $A_{*}$ is equal to $\left\{r \geq 0: r^{2} \in \mathbb{Z}\right\}$ if $d \geq 5$.

In this note we prove an endpoint estimate for the theorem of Magyar, Stein and Wainger. For $p, q \in[1, \infty]$ let $L^{p, q}\left(\mathbb{Z}^{d}\right)$ denote the usual Lorentz space of functions on $\mathbb{Z}^{d}$. We have the following restricted weak type estimate.

Theorem 1. Assume that $d \geq 5$ and let $p_{d}=d /(d-2)$. The discrete spherical maximal function $A_{*}$ extends to a bounded operator from $L^{p_{d}, 1}\left(\mathbb{Z}^{d}\right)$ to $L^{p_{d}, \infty}\left(\mathbb{Z}^{d}\right)$.

The Euclidean analogue of this theorem was proved by Bourgain [1: the Euclidean spherical maximal function $\mathcal{A}_{*}$ extends to a bounded operator from $L^{d /(d-1), 1}\left(\mathbb{R}^{d}\right)$ to $L^{d /(d-1), \infty}\left(\mathbb{R}^{d}\right)$ if $d \geq 3$. This restricted weak type estimate fails in dimension $d=2$ (see [6, Proposition 1.5]).

Our proof of Theorem 1 follows the line of the proof of the theorem of Magyar, Stein and Wainger [5. The main ingredients are the circle method of Hardy, Littlewood and Ramanujan, the Poisson summation formula, and a transference principle. Our simplification is that we will not need the dyadic version of the theorem due to Magyar [4]. Instead, we decompose our operator into an $L^{1}$ part and an $L^{2}$ part depending on a parameter $\alpha$. We use the discrete Hardy-Littlewood maximal function to establish the $L^{1}$ bounds and the error analysis in [5] together with a lemma of Bourgain [1] for the $L^{2}$ bounds.

I would like to thank S. Wainger for several useful discussions on the subject.

\section{Proof of Theorem 1}

We first replace the operator $A_{*}$ with the operator

$$
\widetilde{A_{*}}(f)(n)=\sup _{r \in \Lambda}\left|\frac{1}{r^{(d-2)}} \sum_{|m|=r} f(n-m)\right|
$$

where $\Lambda=\left\{r \in[1, \infty): r^{2} \in \mathbb{Z}\right\}$ and $f: \mathbb{Z}^{d} \rightarrow \mathbb{C}$ is compactly supported. This is possible since $d \geq 5$ and $N_{d}(r) \approx r^{d-2}$. It remains to prove that $\widetilde{A_{*}}$ extends to a bounded operator from $L^{p_{d}, 1}\left(\mathbb{Z}^{d}\right)$ to $L^{p_{d}, \infty}\left(\mathbb{Z}^{d}\right)$. This is an easy consequence of the following lemma.

Lemma 2. For any $\alpha \in(0,1]$ there are two subadditive operators $A_{\alpha}^{1}$ and $A_{\alpha}^{2}$ with the property that $\widetilde{A_{*}}(f)(n) \leq\left|A_{\alpha}^{1}(f)(n)\right|+\left|A_{\alpha}^{2}(f)(n)\right|$ for any $n \in \mathbb{Z}^{d}$,

$$
\left\|A_{\alpha}^{1}(f)\right\|_{L^{1, \infty}} \leq C \alpha^{-2}\|f\|_{L^{1}}
$$

and

$$
\left\|A_{\alpha}^{2}(f)\right\|_{L^{2}} \leq C \alpha^{(d-4) / 2}\|f\|_{L^{2}}
$$

for any compactly supported function $f: \mathbb{Z}^{d} \rightarrow \mathbb{C}$.

The method of proving restricted weak type inequalities by decomposing the operator as in Lemma2 2 is due to Bourgain [1]. An abstract version of this argument may be found in the appendix of [3]. First, we show how to use Lemma2 2 to prove the theorem. By the general theory of Lorentz spaces, the $L^{p_{d}, 1} \rightarrow L^{p_{d}, \infty}$ boundedness of the operator $\widetilde{A_{*}}$ is equivalent to

$$
\left\|\widetilde{A_{*}}\left(\chi_{F}\right)\right\|_{L^{p_{d}}, \infty} \leq C|F|^{1 / p_{d}}
$$


for any finite set $F$, where $\chi_{F}$ denotes the characteristic function of the set $F$ and $|F|$ denotes its cardinality. Clearly, $\widetilde{A_{*}}\left(\chi_{F}\right)(n) \leq C_{d}$ for any $n$, where $C_{d}$ is the constant with the property that $N_{d}(r) \leq C_{d} r^{d-2}$ for any $r \geq 1$. Thus (2.3) is equivalent to proving that for any $\lambda \in(0,1]$,

$$
\lambda^{p_{d}}\left|\left\{n: \widetilde{A_{*}}\left(\chi_{F}\right)(n)>\lambda\right\}\right| \leq C|F| .
$$

By Lemma 2 we have

$$
\begin{aligned}
\left|\left\{n: \widetilde{A_{*}}\left(\chi_{F}\right)(n)>\lambda\right\}\right| & \leq\left|\left\{n: A_{\alpha}^{1}\left(\chi_{F}\right)(n)>\lambda / 2\right\}\right|+\left|\left\{n: A_{\alpha}^{2}\left(\chi_{F}\right)(n)>\lambda / 2\right\}\right| \\
& \leq \frac{2}{\lambda}\left\|A_{\alpha}^{1}\left(\chi_{F}\right)\right\|_{L^{1, \infty}}+\frac{4}{\lambda^{2}}\left\|A_{\alpha}^{2}\left(\chi_{F}\right)\right\|_{L^{2}}^{2} \\
& \leq C \lambda^{-1} \alpha^{-2}|F|+C \lambda^{-2} \alpha^{d-4}|F| .
\end{aligned}
$$

Since $p_{d}=d /(d-2)$, the estimate (2.4) follows by taking $\alpha=\lambda^{1 /(d-2)}$.

It remains to prove Lemma 2, Fix $\alpha \in(0,1]$. We will use some of the notation in [5]. Let

$$
A_{r}(f)(n)=\frac{1}{r^{(d-2)}} \sum_{|m|=r} f(n-m)
$$

and

$$
M_{r}(f)=\sum_{q=1}^{\infty} \sum_{1 \leq a \leq q,(a, q)=1} e^{-2 \pi i r^{2} a / q} M_{r}^{a / q}(f),
$$

where, as in [5], $M_{r}^{a / q}$ is the convolution operator whose multiplier is

$$
\sum_{\ell \in \mathbb{Z}^{d}} G(a / q, \ell) \Psi_{q}(\xi-\ell / q) \widehat{d \sigma}_{r}(\xi-\ell / q) .
$$

Here $G(a / q, \ell)$ is the normalized Gauss sum

$$
G(a / q, \ell)=q^{-d} \sum_{n \in \mathbb{Z}^{d} /(q \mathbb{Z})^{d}} e^{2 \pi i\left(|n|^{2} a / q+n \cdot \ell / q\right)},
$$

$\Psi$ is a smooth cutoff function supported in the cube $Q / 2=\left\{\xi:\left|\xi_{j}\right| \leq 1 / 4, j=\right.$ $1, \ldots, d\}$ and identically equal to 1 in the cube $Q / 4, \Psi_{q}(\eta)=\Psi(q \eta), \widehat{d \sigma}$ is the Fourier transform of the invariant measure on the sphere $|x|=1$ normalized with total measure 1 , and $\widehat{d \sigma}_{r}(\eta)=\widehat{d \sigma}(r \eta)$. The reason for considering the operators $M_{r}$ is that they are good approximations (in $L^{2}$ ) of the operators $A_{r}$. In what follows we assume that $r$ is restricted so that $r \geq 1$ and $r^{2} \in \mathbb{Z}$. Let $N=1 / \alpha \geq 1$. We have

$$
\begin{aligned}
\widetilde{A}_{*}(f)(n) & =\sup _{r}\left|A_{r}(f)(n)\right| \leq \sup _{r \leq 10 N}\left|A_{r}(f)(n)\right|+\sup _{r \geq 10 N}\left|A_{r}(f)(n)\right| \\
& \leq \sup _{r \leq 10 N}\left|A_{r}(f)(n)\right|+\sup _{r \geq 10 N}\left|\left(A_{r}-M_{r}\right)(f)(n)\right|+\sup _{r \geq 10 N}\left|M_{r}(f)(n)\right| \\
& =A_{\alpha}^{1,1}(f)(n)+A_{\alpha}^{2,1}(f)(n)+\sup _{r \geq 10 N}\left|M_{r}(f)(n)\right| .
\end{aligned}
$$

Let

$$
\mathcal{M}(f)(n)=\sup _{r} \frac{1}{r^{d}} \sum_{|m| \leq r}|f(n-m)|
$$


denote the discrete Hardy-Littlewood maximal function. By the same argument as in Euclidean spaces we have

$$
\left\|\left.\mathcal{M}(f)\right|_{L^{1, \infty}\left(\mathbb{Z}^{d}\right)} \leq C\right\| f \|_{L^{1}\left(\mathbb{Z}^{d}\right)} .
$$

We use the operator $\mathcal{M}$ to bound the operator $A_{\alpha}^{1,1}$. We have

$$
A_{\alpha}^{1,1}(f)(n) \leq \sup _{r \leq 10 N} \frac{1}{r^{d-2}} \sum_{|m|=r}|f(n-m)| \leq 100 N^{2} \mathcal{M}(f)(n) .
$$

The desired bound

$$
\left\|A_{\alpha}^{1,1}(f)\right\|_{L^{1, \infty}} \leq C \alpha^{-2}\|f\|_{L^{1}}
$$

follows from (2.5) and the fact that $N^{2}=\alpha^{-2}$.

For the operator $A_{\alpha}^{2,1}$ we use Proposition 4.1 in [5], which can be written in the form

$$
\left\|\sup _{r \in[R, 2 R]}\left|A_{r}(f)-M_{r}(f)\right|\right\|_{L^{2}} \leq C R^{-(d-4) / 2}\|f\|_{L^{2}}
$$

for $R \geq 1$ and $d \geq 5$. Since the supremum in the definition of the operator $A_{\alpha}^{2,1}$ is taken over $r \geq 10 N$, it follows that

$$
\left\|A_{\alpha}^{2,1}(f)\right\|_{L^{2}} \leq C \alpha^{(d-4) / 2}\|f\|_{L^{2}}
$$

as desired.

It remains to decompose the operator

$$
f \rightarrow \sup _{r \geq 10 N}\left|M_{r}(f)(n)\right| .
$$

For this we write first

$$
\sup _{r \geq 10 N}\left|M_{r}(f)(n)\right| \leq C \sup _{r \geq 10 N} \sum_{q=1}^{N / 10} \sum_{1 \leq a \leq q,(a, q)=1}\left|M_{r}^{a / q}(f)(n)\right|+C A_{\alpha}^{2,2}(f)(n)
$$

where

$$
A_{\alpha}^{2,2}(f)(n)=\sum_{q \geq N / 10} \sum_{1 \leq a \leq q,(a, q)=1} \sup _{r}\left|M_{r}^{a / q}(f)(n)\right| .
$$

To bound the operator $A_{\alpha}^{2,2}$ we use Proposition 3.1(a) in [5] for $p=2$ :

$$
\left\|\sup _{r} M_{r}^{a / q}(f)\right\|_{L^{2}} \leq C q^{-d / 2}\|f\|_{L^{2}} .
$$

We can sum this bound over $q \geq N / 10$ and $a \in[1, q] \cap \mathbb{Z}$ to obtain

$$
\left\|A_{\alpha}^{2,2}(f)\right\|_{L^{2}} \leq C \alpha^{(d-4) / 2}\|f\|_{L^{2}}
$$

as desired.

It remains to decompose the operators $M_{r}^{a / q}$ for integers $q \in[1, N / 10]$. For this let $M_{r, \alpha}^{a, q, 1}$ denote the convolution operator given by the multiplier

$$
\sum_{\ell \in \mathbb{Z}^{d}} G(a / q, \ell) \Psi_{q}(\xi-\ell / q) \widehat{d \sigma}_{r}(\xi-\ell / q) \Psi_{r q / N}(\xi-\ell / q),
$$

and let $M_{r, \alpha}^{a, q, 2}$ denote the convolution operator given by the multiplier

$$
\sum_{\ell \in \mathbb{Z}^{d}} G(a / q, \ell) \Psi_{q}(\xi-\ell / q) \widehat{d \sigma}_{r}(\xi-\ell / q)(1-\Psi)_{r q / N}(\xi-\ell / q) .
$$


The notation is, as before, $F_{\lambda}(\eta)=F(\lambda \eta)$. Clearly, $M_{r}^{a / q}=M_{r, \alpha}^{a, q, 1}+M_{r, \alpha}^{a, q, 2}$. Let

$$
M_{\alpha}^{1}(f)(n)=\sup _{r \geq 10 N} \sum_{q=1}^{N / 10} \sum_{1 \leq a \leq q,(a, q)=1}\left|M_{r, \alpha}^{a, q, 1}(f)(n)\right|
$$

and

$$
M_{\alpha}^{2}(f)(n)=\sum_{q=1}^{N / 10} \sum_{1 \leq a \leq q,(a, q)=1} \sup _{r \geq 10 N}\left|M_{r, \alpha}^{a, q, 2}(f)(n)\right| .
$$

This is the decomposition of the remaining operator into an $L^{1}$ part and an $L^{2}$ part.

For the $L^{1}$ estimate we will prove that

$$
\left\|M_{\alpha}^{1}(f)\right\|_{L^{1, \infty}} \leq C \alpha^{-2}\|f\|_{L^{1}} .
$$

For this we need an estimate on the kernel of the operator $M_{r, \alpha}^{a, q, 1}$. Let $K_{r, \alpha}^{a, q, 1}$ denote this kernel. Notice that $\Psi_{q}(\xi-\ell / q) \Psi_{r q / N}(\xi-\ell / q)=\Psi_{r q / N}(\xi-\ell / q)$ since $r \geq 10 N$. Let $Q$ denote the standard cube $Q=\left\{\xi=\left(\xi_{1}, \ldots, \xi_{d}\right):-1 / 2<\xi_{j} \leq 1 / 2\right\}$. Then by letting $\ell=\ell^{\prime}+q s, \ell^{\prime} \in\{0,1, \ldots, q-1\}^{d}, s \in \mathbb{Z}^{d}$ we have

$$
\begin{aligned}
& K_{r, \alpha}^{a, q, 1}(m)=\int_{Q} e^{2 \pi i m \cdot \xi} \sum_{\ell \in \mathbb{Z}^{d}} G(a / q, \ell) \widehat{d \sigma}_{r}(\xi-\ell / q) \Psi_{r q / N}(\xi-\ell / q) d \xi \\
& =\sum_{\ell^{\prime} \in \mathbb{Z}^{d} /(q \mathbb{Z})^{d}} G\left(a / q, \ell^{\prime}\right) \sum_{s \in \mathbb{Z}^{d}} \int_{Q} e^{2 \pi i m \cdot \xi} \widehat{d \sigma}_{r}\left(\xi-s-\ell^{\prime} / q\right) \Psi_{r q / N}\left(\xi-s-\ell^{\prime} / q\right) d \xi \\
& =\left(\sum_{\ell^{\prime} \in \mathbb{Z}^{d} /(q \mathbb{Z})^{d}} G\left(a / q, \ell^{\prime}\right) e^{2 \pi i m \cdot \ell^{\prime} / q}\right) \int_{\mathbb{R}^{d}} e^{2 \pi i m \cdot \eta} \widehat{d \sigma}_{r}(\eta) \Psi_{r q / N}(\eta) d \eta \\
& =e^{2 \pi i|m|^{2} a / q} r^{-d} \int_{\mathbb{R}^{d}} e^{2 \pi i \eta \cdot m / r} \widehat{d \sigma}(\eta) \Psi_{q / N}(\eta) d \eta=e^{2 \pi i|m|^{2} a / q} r^{-d} d \sigma * \psi^{q / N}(m / r) .
\end{aligned}
$$

Here $\psi$ is the inverse (Euclidean) Fourier transform of $\Psi$, the convolution denotes the Euclidean convolution and $\psi^{q / N}(x)=(N / q)^{d} \psi(x N / q)$. Since $\psi$ is a Schwartz function, it is easy to see that

$$
\left|d \sigma * \psi^{q / N}(x)\right| \leq C(1+|x|)^{-(d+1)} N / q
$$

for any $x \in \mathbb{R}^{d}$. Thus,

$$
\left|K_{r, \alpha}^{a, q, 1}(m)\right| \leq C r^{-d}(1+|m| / r)^{-(d+1)} N / q .
$$

By summing this bound over $a \in[1, q] \cap \mathbb{Z}$ and $q \in[1, N / 10] \cap \mathbb{Z}$ we have

$$
\sum_{q=1}^{N / 10} \sum_{1 \leq a \leq q,(a, q)=1}\left|M_{r, \alpha}^{a, q, 1}(f)(n)\right| \leq C|f| * K_{r, \alpha}(n)
$$

where

$$
K_{r, \alpha}(m)=N^{2} r^{-d}(1+|m| / r)^{-(d+1)} .
$$

Thus,

$$
M_{\alpha}^{1}(f)(n) \leq C N^{2} \mathcal{M}(f)(n)
$$

and the estimate (2.9) follows from (2.5).

For the $L^{2}$ estimate we will prove that

$$
\left\|M_{\alpha}^{2}(f)\right\|_{L^{2}} \leq C \alpha^{(d-4) / 2}\|f\|_{L^{2}} .
$$


By the formula of $M_{\alpha}^{2}$, it suffices to prove that

$$
\left\|M_{r, \alpha}^{a, q, 2}(f)\right\|_{L_{L^{\infty}(\Lambda)}} \leq C q^{-1} N^{-(d-2) / 2}\|f\|_{L^{2}}
$$

where $L_{L^{\infty}(\Lambda)}^{2}$ denotes the space of $L^{2}$ functions on $\mathbb{Z}^{d}$ with values in the Banach space $L^{\infty}(\Lambda)$. We first argue as in $[5]$. Let $\widetilde{\Psi}$ be a smooth function supported in $Q$ with the property that $\widetilde{\Psi}(\xi) \equiv 1$ in $Q / 2$. The operator $M_{r, \alpha}^{a, q, 2}$ can be written as the composition of two operators with multipliers

$$
\sum_{\ell \in \mathbb{Z}^{d}} G(a / q, \ell) \widetilde{\Psi}_{q}(\xi-\ell / q)
$$

and

$$
\sum_{\ell \in \mathbb{Z}^{d}} \Psi_{q}(\xi-\ell / q) \widehat{d \sigma}_{r}(\xi-\ell / q)(1-\Psi)_{r q / N}(\xi-\ell / q)
$$

respectively. Let $S^{a, q}$ and $T_{r, \alpha}^{q}$ denote the two operators. Since $|G(a / q, \ell)| \leq$ $C q^{-d / 2}$, we have

$$
\left\|S^{a, q}\right\|_{L^{2} \rightarrow L^{2}} \leq C q^{-d / 2} .
$$

Let

$$
m_{r, \alpha}^{q}(\eta)=\Psi_{q}(\eta) \widehat{d \sigma}_{r}(\eta)(1-\Psi)_{r q / N}(\eta) .
$$

The multiplier $m_{r, \alpha}^{q}$ is supported in $Q / q$. By the transference principle of Magyar, Stein and Wainger (Corollary 2.1 in [5]), we have

$$
\left\|T_{r, \alpha}^{q}\right\|_{L^{2}\left(\mathbb{Z}^{d}\right) \rightarrow L_{L(\Lambda)}^{2}\left(\mathbb{Z}^{d}\right)} \leq C\left\|\widetilde{T}_{r, \alpha}^{q}\right\|_{L^{2}\left(\mathbb{R}^{d}\right) \rightarrow L_{L^{\infty}(\Lambda)}^{2}}\left(\mathbb{R}^{d}\right)
$$

where $\widetilde{T}_{r, \alpha}^{q}$ denotes the operator with multiplier $m_{r, \alpha}^{q}$ acting on functions in $L^{2}\left(\mathbb{R}^{d}\right)$. It remains to prove that

$$
\left\|\widetilde{T}_{r, \alpha}^{q}\right\|_{L^{2}\left(\mathbb{R}^{d}\right) \rightarrow L_{L^{\infty}(\Lambda)}^{2}}\left(\mathbb{R}^{d}\right) \leq C(q / N)^{(d-2) / 2} .
$$

The operator $\widetilde{T}_{r, \alpha}^{q}$ can be written as the composition of two operators with multipliers $\eta \rightarrow \Psi_{q}(\eta)$ and $\eta \rightarrow \widetilde{m}_{r}(\eta)$, respectively, where

$$
\widetilde{m}(\eta)=\widehat{d \sigma}(\eta)(1-\Psi)(q \eta / N)
$$

and $\widetilde{m}_{r}(\eta)=\widetilde{m}(r \eta)$. The operator defined by the multiplier $\eta \rightarrow \Psi_{q}(\eta)$ is bounded on $L^{2}\left(\mathbb{R}^{d}\right)$ uniformly in $q$. Let $\widetilde{U}_{r}$ denote the operator with multiplier $\widetilde{m}_{r}$ acting on functions in $L^{2}\left(\mathbb{R}^{d}\right)$. We will use the following lemma of Bourgain (Proposition 2 in [1]):

Lemma (Bourgain [1). Assume that $m: \mathbb{R}^{d} \rightarrow \mathbb{C}$ is a smooth function and $U_{r}$ is the operator defined by the multiplier $\eta \rightarrow m_{r}(\eta)=m(r \eta)$. Then

$$
\left\|\sup _{r>0}\left|U_{r} f\right|\right\|_{L^{2}} \leq C \Gamma(m)\|f\|_{L^{2}}
$$

for any Schwartz function $f$ where

$$
\Gamma(m)=\sum_{j \in \mathbb{Z}} \alpha_{j}^{1 / 2}\left(\alpha_{j}^{1 / 2}+\beta_{j}^{1 / 2}\right)
$$

with

$$
\alpha_{j}=\sup _{|\eta| \in\left[2^{j}, 2^{j+1}\right]}|m(\eta)| \quad \text { and } \beta_{j}=\sup _{|\eta| \in\left[2^{j}, 2^{j+1}\right]}|\nabla m(\eta) \cdot \eta| \text {. }
$$


In our case, the multiplier $\widetilde{m}$ is supported in the set $|\eta| \geq N /(8 q)$ since $\Psi(\eta)=1$ is $|\eta| \leq 1 / 8$. In addition, $|\widehat{d \sigma}(\eta)| \leq C(1+|\eta|)^{-(d-1) / 2}$ and $|\nabla \widehat{d \sigma}(\eta)| \leq C(1+$ $|\eta|)^{-(d-1) / 2}$. Thus in our case, $\alpha_{j} \leq C 2^{-j(d-1) / 2}$ and $\beta_{j} \leq C 2^{-j(d-3) / 2}$ if $2^{j} \geq$ $N /(16 q)$ and $\alpha_{j}=\beta_{j}=0$ if $2^{j}<N /(16 q)$. Thus $\Gamma(\widetilde{m}) \leq C(q / N)^{(d-2) / 2}$. By Bourgain's lemma,

$$
\left\|\widetilde{U}_{r}\right\|_{L^{2}\left(\mathbb{R}^{d}\right) \rightarrow L_{L}^{2}\left(\mathbb{R}_{+}\right)}\left(\mathbb{R}^{d}\right) \leq C(q / N)^{(d-2) / 2} .
$$

This proves (2.14). The estimate (2.11) follows from (2.12), 2.13) and (2.14), and the estimate (2.10) follows by summing over $q$ and $a$.

We can now finish the proof of Lemma 2. Let $A_{\alpha}^{1}(f)=A_{\alpha}^{1,1}(f)+M_{\alpha}^{1}(f)$ and $A_{\alpha}^{2}(f)=A_{\alpha}^{2,1}(f)+A_{\alpha}^{2,2}(f)+M_{\alpha}^{2}(f)$. The estimate (2.1) follows from (2.6) and $(2.9)$, and the estimate (2.2) follows from (2.7), (2.8) and (2.10).

\section{REFERENCES}

[1] J. Bourgain, Estimations de certaines fonctions maximales, C. R. Acad. Sci. Paris 301, Série I (1985), 499-502. MR 87b:42023

[2] J. Bourgain, Averages in the plane over convex curves and maximal operators, J. Anal. Math. 47 (1986), 69-85. MR 88f:42036

[3] A. Carbery, A. Seeger, S. Wainger and J. Wright, Classes of singular integral operators along variable lines, J. Geom. Anal. 9 (1999), 583-605. MR 2001g:42026

[4] A. Magyar, $L^{p}$-bounds for spherical maximal operators on $\mathbb{Z}^{n}$, Rev. Mat. Iberoamericana 13 (1997), 307-317. MR 99d:42031

[5] A. Magyar, E. M. Stein and S. Wainger, Discrete analogues in harmonic analysis: Spherical averages, Ann. of Math. 155 (2002), 189-208. MR 2003f:42028

[6] A. Seeger, T. Tao and J. Wright, Endpoint mapping properties of spherical maximal operators, J. Inst. Math. Jussieu 2 (2003), 109-144.

[7] E. M. Stein, Maximal functions I: Spherical means, Proc. Nat. Acad. Sci. 73 (1976), 21742175. MR 54:8133a

Department of Mathematics, University of Wisconsin at Madison, Madison, WisconSIN 53706

E-mail address: ionescu@math.wisc.edu 